\title{
Entrevista con Manuel Antonio Garretón: "La nueva Constitución va a definir la superación del orden neoliberal y una nueva relación entre política y sociedad" 1
}

\author{
Interview with Manuel Antonio Garretón: "The new Constitution will define the \\ overcoming of the neoliberal order and a new relationship between politics and society"
}

\author{
Alejandro Osorio Rauld (Universitat d'Alacant, España) \\ Octavio Avendaño (Universidad de Chile, Chile)
}

\begin{abstract}
Cita bibliogràfica: Osorio Rauld, A. y Avendaño, O. (2022). Entrevista con Manuel Antonio Garretón: “La nueva Constitución va a definir la superación del orden neoliberal y una nueva relación entre política y sociedad”. Disjuntiva, 3(1), 55-62. https://doi.org/10.14198/DISJUNTIVA2022.3.1.5
\end{abstract}

La entrevista que acá presentamos fue realizada en el contexto de creación de la Convención Constitucional, actor institucional que redactará entre este año y el siguiente una nueva Constitución para Chile. Para indagar con mayor profundidad en este fenómeno de nuevo ciclo político que se inicia en el país sudamericano, decidimos conversar con el académico y Premio Nacional de Ciencias Sociales y Humanidades, Manuel Antonio Garretón. Ello porque su trabajo intelectual, validado en todo el mundo, ha consistido en toda una vida de esfuerzo dedicada al estudio de los procesos sociales, políticos y económicos en América Latina, con especial énfasis en el caso chileno. Su aporte al campo de las ciencias sociales latinoamericanas ha sido fundamental para pensar la articulación entre política y sociedad en la región, siendo este conocimiento fundamental para comprender hoy la especificidad de un conjunto de revueltas sociales en Latinoamérica, lo que podría augurar una nueva era política que se iniciaría con el estallido social y el proceso constituyente chileno.

El diálogo con el académico estuvo centrado fundamentalmente en dos tópicos: un primer eje centrado en los antecedentes y la naturaleza del estallido social del día 18 de octubre de 2019 y un segundo eje enfocado en la comprensión de la respuesta o output que produce la clase política para responder a las demandas del mundo social. En ella, el autor nos advierte de la existencia de un nuevo tipo de relación entre la élite política y la ciudadanía, distinta a la imbricación entre ellas que ha marcado la historia del país, ello en el contexto de un escenario complejo e incierto, sobre todo, porque aún no hay indicios claros respecto al nuevo tipo de institucionalidad que se elaborará en la nueva Constitución.

1. Un extracto de esta entrevista fue publicado en una versión sintetizada en la Revista de Ciencias Sociales Ambos Mundos n². En esta nueva versión extendida hemos incorporado material inédito referido a la dificultad de los partidos de oposición para enfrentar el nuevo escenario político y también sobre el actuar del gobierno del presidente Piñera en el contexto de la pandemia, donde se analiza cómo su actuación pudo haber condicionado negativamente el apoyo a la derecha en la Convención Constitucional.

Manuel A. Garretón: https://orcid.org/0000-0002-7062-675X

Octavio Avendaño: https://orcid.org/0000-0001-6945-5327

Alejandro Osorio: https://orcid.org/0000-0003-0409-0376

Correo electrónico de correspondencia: alejandro.osorio@ua.es; oavendanop@u.uchile.cl 
Alejandro Osorio (AO): En 2019 Chile se encontraba en un contexto de relativa normalidad antes del estallido social, se decía que el país iba progresando bien en relación al resto de países de la región. Lo puedo decir con cierta certeza, ya que en el contexto de una investigación ${ }^{2}$ me tocó entrevistar a varios líderes empresariales en 2019 quienes, en su opinión, veían que Chile iba bien encaminado. Sin embargo, en octubre de ese año se produce un estallido social cuyos motivos aún carecen de cierto consenso. En ese sentido, nos gustaría partir preguntando ¿cuáles, a tu juicio, son los principales factores que detonan dicho acontecimiento?

Manuel Antonio Garretón (MAG): Yo creo que hay que ubicar el estallido antes de entrar a sus particularidades, en una especie de "espíritu" de la época, que se da en todo el mundo y se da con algunas particularidades en América Latina y en especial en Chile. Y eso está expresado en tipos de revueltas que son parecidas en todas partes. Estas apuntan a una crisis muy compleja de la idea de sociedad; una sociedad "estallada". Pienso, por ejemplo, en los efectos de la revolución digital, lo que hace que la idea clásica de polis de alguna manera se vaya perdiendo y lo que importa es el modo acerca de cómo "yo me relaciono y resuelvo mis problemas" o "me resuelven mis problemas". En tal sentido, vemos que la ciudadanía es mucho más un agregado de colectividades, de identidades particulares, de individuos e individuas, de territorios (que es un concepto que aparece mucho en el último tiempo), que la idea tradicional de una polis, territorio y población que tiene una economía, una política, una cultura o varias culturas, una forma de organización social y un centro de toma de decisiones que es el Estado.

Existe el imperativo de que la democracia y la política resuelvan los problemas de la gente, pero interesa menos que resuelvan los problemas de la colectividad. Eso ha estallado y de alguna manera estalla en todas partes del mundo, lo que trae consigo una crisis muy profunda, que para algunos autores sería una "crisis terminal" de la democracia representativa. Ciertamente, hay una crisis de la representatividad y eso creo que es muy importante tenerlo en cuenta a la hora del análisis.

Hay que agregar a lo anterior, nuevas formas de convivencia, que tienen que ver con las redes sociales, que enfatizan el aspecto de los intereses, ya sea de comunicaciones individuales (yo me comunico como quiero, a la hora que quiero y digo lo que quiero, si quiero escucho y si quiero no), pero también como "redes" que pueden producir acuerdos de acciones inmediatas.

Uno no puede dejar de entender a cabalidad el concepto de "estallido" sin tener en cuenta estas nuevas formas de convivencia.

Ahora, si consideramos la particularidad del caso chileno, hay que señalar un ciclo de movilizaciones: en 2006 con los estudiantes secundarios y trabajadores subcontratistas; en 2011-2012, con estudiantes universitarios y otras de carácter medio ambiental, territoriales, pueblos originarios; y en 2018, principalmente, feministas. Se trata de las primeras grandes movilizaciones que se hacen fuera de los partidos, por tanto, fuera del liderazgo político institucional, aunque en algunos casos son organizaciones formales representativas como las federaciones de estudiantes.

Pese a las distintas opiniones que hay, he sostenido que las manifestaciones en Chile, incluyendo las de la época más dura de la dictadura, siempre estuvieron imbricadas con partidos políticos y la mayor parte de las veces lideradas por dirigentes políticos. Estos actores no necesariamente pertenecían a una cúpula, pero eran dirigentes intermedios vinculados a partidos políticos. Hay una cosa interesante que se produce en la época de la dictadura: el surgimiento de una franja constituida entre militante y activista social, que es la que mantiene la continuidad de la relación entre partidos políticos y ciudadanía, de modo que, en el momento en que los partidos tienen que actuar y tener una posición, una estrategia y convocar, esta franja aseguraba que la gente respondiera vía movilización. El mejor ejemplo de esto es el plebiscito de 1988, que pone fin a la dictadura militar, cuya participación electoral fue sobre el 97\%. En contraste, en las movilizaciones de 2011-2012 ya no intervienen los partidos de la misma forma y ya no poseen capacidad de convocatoria. Y en el estallido de 2019 ya no hay tampoco grandes organizaciones clásicas liderándolas.

Pero no sólo las particularidades de las nuevas formas de movilización deben ser señaladas, sino también el contenido de ellas. Las movilizaciones del estallido de 2019 marcan el surgimiento de un proyecto histórico político distinto a lo que se ha denominado la "transición", entendida como término de la dictadura y restitución de la democracia. Y en el fondo, el eje de las reivindicaciones de la ciudadanía apunta al término del modelo socioeconómico neoliberal y su cristalización institucional en la Constitución de 1980.

2. La investigación mencionada es un artículo sobre la postura política de los grandes empresarios chilenos frente al cambio de la Constitución poco antes del estallido social. En este trabajo se demuestra que la élite empresarial del país sudamericano rechazaba un cambio de la Constitución, entre otras razones, por considerar que el desarrollo socioeconómico iba, a su parecer, en el camino correcto, por lo que no se hacía necesario introducir cambios constitucionales. Al respecto, véase Osorio y Reyes (2021). 


\section{Octavio Avendaño (OA): En tu opinión ¿existe alguna relación entre la Constitución de 1980 modificada parcialmente por los gobiernos de la Concertación y el estallido social?}

MAG: Pienso que la Constitución de Pinochet tiene que ser vista como la proyección institucional del bombardeo a la Moneda y este fenómeno desata todas las memorias, las subjetividades y las reivindicaciones ideológicas de la gente en forma permanente. Era una Constitución que careció siempre de legitimidad, no solo de origen, sino valórica, y lo que podía existir era una escueta legitimidad instrumental; es decir, se reconocía que estaba ahí, que había que aplicar, pero, "estando en contra de ello".

Al respecto, el movimiento estudiantil entre 2011-2012 lo que plantea como primera reivindicación es educación pública de calidad, lo que significa revindicar el papel del Estado y, en términos más profundos, el cambio del modelo cultural. La segunda reivindicación es la reforma tributaria. Se decía en aquella época que era para garantizar recursos que permitieran hacer la reforma educacional. Sin embargo, no se trataba sólo de eso, pues la reforma tributaria era un fin en sí mismo, su objetivo era mayor redistribución económica y de igualdad. Y como ningún cambio significativo se podía materializar con la institucionalidad vigente, una nueva Constitución aparece como reivindicación ineludible. Los actores políticos, en especial, la Concertación de Partidos por la Democracia, convertida ahora en Nueva Mayoría con la incorporación del Partido Comunista, en 2013 transforma esas reivindicaciones en un programa de gobierno, intentando responder a la ciudadanía con acción política. Por primera vez en la historia de Chile un proyecto histórico que no venía de la clase política, aunque elaborado por ella, se devuelve a la ciudadanía, en términos de programa de gobierno en una elección como la del 2013 en que participó menos del 50\% del electorado, lo que puso en evidencia la ruptura entre política y ciudadanía.

Entonces, tenemos como un antecedente del estallido un proyecto refundacional con el segundo gobierno de Bachelet -que no se encuentra con la política institucional y que después se empantana- que recogía las aspiraciones colectivas de las movilizaciones de 2011-2012 y que realizó eventos tan importantes como los cabildos y un proceso constituyente informal, que era un intento de movilización y de restituir el lazo entre política y sociedad. Pero esto no tuvo efectos institucionales, y las reformas fundamentales, algunas se cumplieron mientras otras quedaron a medio camino. A su vez, el bloque político que había liderado lo que se llama la transición culminó su descomposición.

El nuevo gobierno de derecha encabezado por Piñera (2018-2022) agravó dos crisis de legitimidad social. En primer lugar, la del modelo socioeconómico neoliberal implantado por la dictadura, corregido, pero no superado por los gobiernos de la Concertación, que había pasado de una especie de legitimidad "fáctica", algunos dirán que incluso valórica debido a que el individualismo se había metido en el corazón y cabeza de la gente, a una creciente deslegitimación. Esta ilegitimidad tenía que ver, entre otras cosas, con el endeudamiento de la sociedad chilena, las promesas incumplidas, el mismo triunfo político de la derecha en el gobierno y, además, porque la concentración de poder económico y las desigualdades eran demasiado evidentes. No se cumplen las promesas de crecimiento económico y menos se cumple la promesa de que la gente sienta que vive y seguirá viviendo mejor, más allá de sus propios esfuerzos. Todo ello, finalmente, va socavando la escasa legitimidad del modelo neoliberal.

\section{AO: ¿Y cuál será el papel de la nueva Constitución en la crisis de legitimidad?}

MAG: Efectivamente, la segunda crisis de legitimidad tiene que ver precisamente con la Constitución establecida en la dictadura (corregida parcialmente por los gobiernos de la Concertación) y con el modelo socioeconómico neoliberal y político que esta consagra. En realidad, esta era una crítica "antigua" que ya venía desplegándose desde el "Grupo de los 24 " a mediados de la década de los 80 . Sin embargo, lo interesante es que esta crítica ahora va a convertirse en una especie de denominador común que agrupa todos los descontentos, por las distintas razones que he señalado. El tema de la nueva Constitución lo van a poner los actores políticos institucionales deslegitimados ante la mayoría de la población (según todos los estudios de opinión pública). O por lo menos ese es el discurso general que se ha internalizado, es un "no creemos a la política institucional" y, en contraste, se produce una suerte de creencia de que la política ya no pertenece a los políticos profesionales, sino a la ciudadanía misma y eso se expresa en "la calle", en movilizaciones, en un proceso sin política institucional o partidaria. Y, sin embargo, será a través de un acuerdo político institucional que se ofrecerá la posibilidad de salida de la crisis de la que era expresión el estallido social.

Así, respecto de la salida de la crisis, la nueva Constitución se va a definir en torno a los dos grandes problemas actuales de la sociedad chilena: la superación del actual orden socioeconómico y el establecimiento de una nueva relación entre política y sociedad. El proceso constituyente es refundacional en esa doble dimensión.

Respecto de las relaciones entre política y sociedad, como sabemos, en Chile, el sujeto social en los últimos 100 años era partido $y$ movimiento, distinto de las experiencias de algunos países del partido-movimiento. En el caso chileno, hablamos 
más bien de partidos y movimiento social de manera imbricada. Los movimientos se movilizaban a través de los partidos políticos, sin que ninguno de estos actores perdiera su autonomía. Los ejemplos clásicos son los movimientos de trabajadores y estudiantiles. De hecho, es bien sabido que en Chile entre 1967 y 1972 se sindicalizaron alrededor de 400.000 campesinos, y eso en cualquier país del mundo habría dado origen a un "partido campesino". No obstante, en Chile, cada partido, incluida la derecha, tenía una federación y entonces los campesinos sindicalizados se incorporaban a esas federaciones vinculadas a partidos sin perder la autonomía de acción. Ello es un buen ejemplo de lo que llamamos imbricación. También lo es la naturaleza del movimiento estudiantil universitario desde los años treinta hasta la década del 2010. Es decir, el modo de incorporación a la sociedad era a través de un modelo político institucional y cuando este no lo permitía se producían nuevas movilizaciones, exigencias de reformas y entonces se volvía a generar una nueva forma de articulación entre partido y sociedad.

En las movilizaciones del estallido, por supuesto, hay grupos esporádicos que se juntan y que organizan diversos tipos de acción colectiva, pero lo sustantivo es que no hay un liderazgo político como antaño vinculado a las organizaciones de trabajadores, pobladores, estudiantes y otros sectores sociales. Tampoco un liderazgo de organizaciones estudiantiles como lo hubo en las movilizaciones de 2006 y 2011, para citar sólo algunos ejemplos. Ahora no hay interlocutor y entonces esa es la expresión más clara, yo diría, de la ruptura de lo que había sido el modelo de relaciones entre la política institucional y los actores sociales, que era lo que llamábamos el modelo de imbricación entre partidos y movimiento, con autonomías relativas de cada uno, pero con autonomías.

Lo que ocurre, entonces, es que esta relación de imbricación se rompe definitivamente, y creo que difícilmente se va a recuperar. Entre otras cosas porque, con el escenario así abierto en la Convención Constitucional, no sabemos bien qué va a ser lo "político institucional". Mi sospecha es que entre lo político institucional y lo social no se va a repetir lo que existió anteriormente en Chile. En el momento del estallido social, el sujeto que se movilizaba en las calles era muy diverso, con una suma de demandas distintas. Por ende, no había originalmente un pliego ni tampoco interés en que existiese uno. Esto hace que el estallido sea vivido como una "revolución" por quienes salieron a las calles a movilizarse, a organizarse de manera espontánea. De alguna manera fue experimentado como una revolución.

OA: Pero si tomamos en cuenta la idea de "irresistibilidad" que propone Hannah Arendt (2001: 223), cuando se refiere a las revoluciones, en donde los movimientos populares adquieren una dinámica propia, sin existir alguien que los conduzca, ¿podríamos considerar al estallido social de octubre en esos términos, más que meros episodios de protesta y de insurrección popular?

MAG: Pienso que estamos más cerca de la "insurrección” o más cerca de la "rebelión” que, de la revolución, porque aquí no hubo toma del poder del Estado. Fuera de esta distinción analítica y para aclarar este punto, creo que la gente en Chile vivió el estallido social como una "revolución”. Entonces, podríamos decir que fue una rebelión que la gente experimentó como revolución, que es lo importante. Pero, considerando la naturaleza de la pregunta, lo importante a señalar es que la gente no aceptó la representación para los cambios de poder, porque, en definitiva, no aceptaba la idea de representación. Por lo tanto, vive la revolución como un acto cotidiano, eso es lo que explica por qué se prolonga tanto en el tiempo y por qué la solución política que es el "Acuerdo Nacional por la Paz Social y la Nueva Constitución”, firmado el 15 de noviembre, es rechazado en las calles. Recordemos que Beatriz Sánchez (candidata presidencial de la izquierda en las anteriores elecciones presidenciales) y Gabriel Boric (actual candidato presidencial de la izquierda) son criticados públicamente por amplios sectores movilizados.

OA: El acuerdo del 15 de noviembre viene a representar un momento de inflexión de esta dinámica de movilización que se estaba llevando a cabo, porque, como bien expusiste, la movilización era "espontánea" en todo el país, no solo en el Gran Santiago. Y en el resto del país, el estallido se vivía de la misma forma, por el hecho de que no había conducción política. En ese sentido, el "Acuerdo" además de lo que va a implicar desde el punto de vista de acercar posiciones entre una parte importante de la oposición y el gobierno, ¿crees tú que también es una oportunidad que va a tener la clase política para lograr conducir esa movilización, o darle una expresión política a toda esa movilización?

MAG: No puede hablarse de los actores políticos como si fueran uno solo y no múltiples y muy diversos. Hay que recordar que el presidente Piñera dijo "guerra", lo que fue desmentido inmediatamente por el jefe de la Defensa Nacional de la Región Metropolitana. Tampoco es posible que movilizaciones como estas puedan ser conducidas. Es cierto que, en una crisis de esas características, algunos buscan frenar lo que está ocurriendo y otros entienden que hay un mensaje potente más allá de las expresiones ideológicas, de los letreros y de las consignas. Hay un significado muy profundo, y yo creo que ahí comienza una nueva época de relaciones entre lo político y lo social que no sabemos cuánto va a durar.

Se inaugura una etapa en la cual la iniciativa parte del mundo social, pero que ya no es la base social clásica de la matriz sociopolítica anterior, basada en las clases sociales o en "fracciones de clase". Ahora lo que observamos es una "base 
social" formada por identidades, individuos y colectivos orientados por principios identitarios, algunos con componentes de transformación universal como es, por ejemplo, el feminismo. Es un mundo social complejo enteramente distinto al anterior (que algunos han llamado el "nuevo pueblo"). Esa gente, ese pueblo movilizado, esa diversidad de actores, es el que ganó las elecciones convencionales de mayo 2021. Ese es el dato central: si la Constitución del 80 fue la proyección institucional del bombardeo a La Moneda, del intento de asesinato que lleva a la muerte de Allende, de la destrucción del sistema institucional chileno, la elección de convencionales de mayo 2021, es la proyección institucional electoral del estallido, es la gente del estallido y de los partidos que apoyaron lo que está representado en la Convención Constitucional.

Ante la crisis, el estallido y las movilizaciones, el mundo político institucional desde el gobierno a la oposición, entiende que estos eventos y procesos estaban por encima de ellos, los habían sobrepasado. Y esto plantea el problema de cómo se puede dialogar con eso. Pues bien, lo que hace la clase política, es ofrecer una "solución institucional" que involucra al sujeto movilizado en la elaboración de una Constitución que reemplace a aquella que la gente rechaza.

Pero lo más relevante es que, como les señalaba, lo que se ha venido produciendo es una nueva manera de vinculación entre lo político y lo social, lo que implica que los actores políticos adopten distintas posiciones frente a esta solución de la crisis. Por ejemplo, ya sabemos que el Partido Comunista y parte del Frente Amplio se opusieron al Acuerdo del 15/N porque pensaban que eso era darle una oportunidad al gobierno de Piñera, otorgando así una suerte de "salvavidas" al presidente. Sin embargo, considero incorrecta esa interpretación. El Acuerdo del 15/N fue el encauzamiento institucional para precisamente responder a las demandas del movimiento social, fue diseñado para eso. Hubo una movilización de la ciudadanía sin el mundo político institucional, pero con respuesta de este, aunque sin consultar antes a la ciudadanía. Esto genera un rechazo inicial al Acuerdo de los actores político partidarios, pero luego se produce una aceptación del contenido de la propuesta, aunque posteriormente se vuelve a esta desafección y rechazo hacia aquellos.

En el fondo, lo que hay que entender es que el Acuerdo del 15/N no implica "imbricación” como he comentado aquí. Al contrario, aquí hubo ruptura de la imbricación. A diferencia de otros países de la región, como es, por ejemplo, entre otros, el caso peruano, en Chile tardó mucho en manifestarse el rechazo a la política institucional. No obstante, después del estallido social es impresionante ver cómo se ha ido potenciando la crítica radical y visceral al mundo de lo político institucional. Sin embargo, en medio de las protestas sociales, el mundo político responde autónomamente, tanto así que no consultó a la ciudadanía en su decisión y eso generó molestia en la gente, y por eso la sociedad responde rechazando aún más a los actores políticos, aunque estos, a su vez, involucran a la ciudadanía para la resolución de la crisis, y es la ciudadanía la que otorga también autónomamente legitimidad a la salida. Esto, a mi juicio, es una forma de hacer política enteramente distinta a la que conoció Chile en los últimos 100 años.

AO: ¿Puede haber influido la pandemia en la escisión entre movimiento y el Acuerdo? Vale la pregunta porque la pandemia y la inacción del gobierno también genera distanciamiento y eso después se expresa en los resultados de la elección de los representantes a la Convención y en el resto de los comicios, de gobernadores y municipales. Previo a todo eso, el gobierno no toma ninguna decisión, dejando a las personas teniendo que enfrentar los efectos de la pandemia con sus propios ahorros previsionales. Es decir, si en algún momento hubo imbricación entre política y sociedad, luego se vuelve a generar distanciamiento.

MAG: Existe un principio metodológico que habría que utilizar, en la medida en que no somos biólogos, ni tampoco especialistas de las ciencias médicas o de las ciencias naturales. Podemos analizar los fenómenos biológicos en términos de qué condiciones sociales pueden haberlo producido y de qué manera responde la sociedad. La pandemia COVID-19 no se puede analizar, en las ciencias sociales, en términos de qué le pasa al virus, sino de qué le pasa a la sociedad. Eso es lo que sabemos hacer y, por lo tanto, nuestro principio es que no puede analizarse la pandemia si no es en relación al contexto económico, social, político y cultural de un país. No puedes analizar lo que ha pasado en la pandemia en Brasil si no consideras el "factor Bolsonaro", un liderazgo que niega el fenómeno mismo. Entonces, en el caso chileno, esto se da en el contexto planteado por el estallido social y por la solución institucional, el encauzamiento de eso en el proceso constituyente. En suma, es necesario analizar lo que ha pasado con la pandemia en ese contexto.

Y aquí hay dos elementos claves: tenemos un gobierno que ya había perdido la capacidad de conducción y la capacidad de entender lo que pasa en la sociedad y que va a recurrir a aquello que sabe hacer, que es mantener una tensión latente, hasta que salga lo de los tres retiros de fondos previsionales ${ }^{3}$, que a la vez combinan estallido, pandemia y el tema de las

3. El entrevistado hace referencia a la política aprobada tres veces por el Congreso Nacional de retirar los fondos previsionales de los afiliados para paliar los efectos económicos de la pandemia. 
pensiones. El gobierno reacciona primero con una posición autoritaria, lo único que le importa es el orden público, a cualquier precio, que se mantenga la capacidad de crecimiento y que el Estado no debe meterse. Después de eso va a ir cambiando su postura. Así, llega tarde y mal, resolviendo los problemas de manera parcial y siempre con "letra chica", lo que es propio de la política focalizada. Después de un primer momento de incertidumbre, interesante es la posición que asume la oposición, que va a criticar la postura inicial del gobierno y respaldar lo que signifique soluciones de emergencia, pero planteando los problemas indicados. Ahí destaca el acuerdo que se hace con los economistas transversales, un acuerdo convocado por la presidenta del Colegio Médico. El gobierno se había aferrado a lo económico y a la idea de no romper con la lógica de focalización de los recursos del Estado. Las soluciones eran con los recursos de la misma gente, por ejemplo, la utilización de los dineros del fondo de cesantía ${ }^{4}$. En general, con la excepción de la política de vacunas, camas de urgencia y ventiladores, el gobierno se mostró incompetente y poco empático con los actores sociales.

Por otra parte, no hay que olvidar que se trata del presidente de un gobierno que además en ese momento estaba impregnado de una ortodoxia absoluta en la manera de resolver el problema de los recursos, es decir, absolutamente contrario a darle una mayor capacidad de acción y de disposición de recursos al Estado para así entregar una renta básica universal. Se daba asimismo un chantaje permanente de la UDI, el partido de la coalición de gobierno más conservador y más ligado a la dictadura en el pasado. Y será justamente este partido el que pagará un precio enorme en las elecciones de convencionales y también en las de alcaldes y concejales. Eso fue lo que sucedió por el lado del gobierno.

Por el lado de la sociedad, habría que ligar el tema de la negativa percepción de la gente en el último año respecto del gobierno y el tratamiento de la pandemia, con la votación de constituyentes en mayo 2021. No tanto con las otras elecciones, como las de alcalde y concejales, dado que ellas obedecen a parámetros clásicos, en donde hay oficialismo y oposición, y en cada uno de estos bloques existen distintos partidos. El paradigma de estas elecciones es distinto a una elección como la de convencionales constituyentes, que se hace por primera vez, en directa conexión con lo que fue el estallido, con paridad de hombres y mujeres, con escaños reservados para pueblos originarios y con independientes reconocidos como tales, para una sola tarea que es redactar la Constitución. Esta elección, la de convencionales, expresa el estallido y, además, el estado de ánimo de la sociedad respecto a lo que ha sido el gobierno y sus partidos, lo que hace que ambos tengan una derrota enorme en comparación a otras elecciones, pues hubo un juicio de la ciudadanía respecto del gobierno en la pandemia. El juicio de la sociedad antes de la pandemia fue el estallido y el juicio de la sociedad sobre el manejo de la pandemia se expresaron en el resultado de la elección de convencionales.

\section{OA: ¿Qué importancia tiene el fenómeno del antipartidismo en el último tiempo y qué implicancias podría llegar a tener en este momento donde precisamente lo que se requiere es asegurar solidez institucional? Estamos viendo que el escenario cambia todas las semanas y a veces en la semana cambia día a día, y eso da cuenta que hay un problema de solidez institucional y los partidos no están reaccionando de manera adecuada, y más encima existe un antipartidismo exacerbado que a veces es generado por partidos y organizaciones de izquierda.}

MAG: Efectivamente, ese es un problema fundamental, pero mi impresión es que esto puede ser más profundo, en el sentido que alguien podría decir, "los partidos están actuando como pueden actuar". Vivimos en situaciones, como dices, en que pareciera que todo es líquido. En realidad, sería mejor decir es viscoso, porque lo viscoso no se escurre totalmente, se pega un poco y es opaco. A mi juicio es más fangoso que "líquido", no fluye fácilmente. También hay algo de estancamiento, porque si fuera todo líquido ya habrían desaparecido los partidos. Hay un problema de aceleración de tiempos para tomas de decisiones. Los fenómenos sociales te llevan a que en pocos días tienes que tomar una decisión a partir de algún hecho que ha provocado un cambio drástico en, llamémoslo para usar los viejos términos, la "correlación de fuerzas", ¿qué tiempo tienen los partidos de consultar a la ciudadanía? Entonces sus decisiones son vistas como lejanas o incomprensibles por la propia ciudadanía.

En el caso chileno los partidos están representando muy poco, aunque hayan usado todas las fórmulas posibles: hacer acuerdos, deshacer acuerdos, rehacer acuerdos. Ahora, por otro lado, si se dice que solo los independientes, o que la mayor parte de diputados o senadores del mundo político institucional debieran ser independientes, se rompe definitivamente el principio de representación porque nadie puede controlar al que fue elegido por cien mil electores, pero que no tiene que rendirle cuentas a nadie, debido a que no tiene compromiso con alguna organización partidaria. De manera que estamos frente a un problema de enorme profundidad porque el tipo de organización a través de partidos, vinculado a clases o relacionado con movimientos, tal como se conoció en los últimos dos siglos, ya no funciona; y eso significa que hay una crisis de la idea de representación, lo que hace necesario reconocer esta pérdida de legitimidad.

4. El entrevistado se refiere a los dineros del seguro de desempleo de los trabajadores como la solución política individual para paliar los efectos económicos de la pandemia. 
Es bien interesante, por ejemplo, que, en el mundo de la política tradicional, el centro político no es tan gravitante como era antes. Eso ya lo había anunciado la derrota de la candidata demócrata cristiana, Carolina Goic, en las elecciones de 2017. En toda su historia, nunca un candidato presidencial demócrata cristiano o apoyado por la DC había sacado un 6\%. El otro problema es cómo se van a conectar los convencionales con el resto de las autoridades, en las que predominan los actores de la política tradicional. Habrá momentos donde esos actores se puedan encontrar y seguro que otros no. De hecho, se vienen elecciones presidenciales en las que pareciera tendrán poco que ver con la elección de convencionales. Entonces, van a coexistir políticas institucionales junto con una profunda recomposición del sistema político. Además, el proceso constituyente ofrece una posibilidad, que es cómo se relaciona la Convención Constitucional con el resto de la ciudadanía; con los plebiscitos y cabildos realizados previamente y con aquellos que vendrán. La pregunta es cómo se va a institucionalizar eso, porque ahí está el embrión, no solo del modelo de sociedad que va a tener que ser implementado a lo largo de muchos años, sino el modo de relación con la política.

\section{AO: Entonces, ¿la Convención Constitucional puede aportar en resolver esta crisis de relación entre política y sociedad?}

MAG: En este sentido, el caso chileno tiene una oportunidad increíble que no la tienen otros países, pues en otros lugares la fórmula son las elecciones -y nada más-, y a través de elecciones se va resolviendo sólo parcialmente ese problema. En cambio, en Chile, se está discutiendo sobre los principios por primera vez a través de una Constitución hecha por el conjunto del país, por la ciudadanía. Fíjense que de alguna manera la idea del Estado plurinacional, que al parecer se impondrá, implica tensionar la idea misma de Estado, el modo como se conciben la República y el Estado. La nueva Constitución va a tener que pronunciarse sobre la crisis medioambiental y sobre la revolución científico-tecnológica. Por citar un solo ejemplo, se sabe que hay un grupo de gente que está trabajando en los derechos neuronales, vale decir, el derecho a la privacidad de tu cerebro. Se está hablando de eso, y será quizás la primera Constitución, a nivel mundial, que plantee ese problema. Será, de seguro, una Constitución que afirmará el principio paritario en todos los niveles, lo que implica ir más allá de la equidad de género. Por tanto, tenemos un país constituyente en este momento -universidades, centros de pensamiento, medios de comunicación, organizaciones territoriales, sociales, gremiales, etc. dedicados a colaborar con la Convención- y ello tanto en los temas de contenido como en los de relación entre política y sociedad.

En tal sentido, estamos en algo parecido, análogo, a lo ocurrido hace poco más de 100 años, en 1920, en donde también hubo un "estallido" que fue la elección de Arturo Alessandri, que implicaba la posibilidad de fin del régimen oligárquico del siglo XIX. En efecto, hubo una Constitución en 1925 que intentó precisamente encauzar la ruptura de lo que había sido el Estado oligárquico, por lo tanto, que permitió una nueva forma del poder político. Había la idea de asambleas provinciales, de "territorios", aunque todavía en un Estado unitario y ellas posteriormente no tuvieron realización. Las formulaciones de la Constitución de 1925 cristalizan de modo puramente político en 1932 (en la segunda administración de Arturo Alessandri) después de varios momentos de inestabilidad antes y después de la dictadura de Ibáñez (1927-1931) y se afirman socialmente con la elección de Pedro Aguirre Cerda (1938). En la actualidad, las tradicionales elecciones presidenciales y parlamentarias son fundamentales para asegurar que lo que pase en la Convención Constitucional empiece a cristalizarse y no haya que esperar 13 años de inestabilidad, como en aquella época. Las transformaciones del orden socioeconómico y político van a madurar probablemente en una o dos décadas más, pero tienen que empezar a implementarse desde ahora, y si no vamos a volver a tener estallido y esta vez sin esperanzas.

OA: De hecho, la Constitución de 1925 demoró alrededor de 7 u 8 años en lograr implementarse sin que generara estabilidad. El período 1931-1932 fue de años caóticos, de conflictos, de caída de gobiernos; luego, vuelve Alessandri en el año 1932, pero utilizando fórmulas que el propio Ibáñez (1927-1931) tuvo que utilizar para poder mantener la estabilidad y asegurar el arraigo de la constitución de 1925. Entre 1932-1938, Alessandri gobierna con "facultades extraordinarias", así como también posteriormente Gabriel González Videla (1946-1952) y Carlos Ibáñez en su segundo gobierno (1952-1958). Esto también ocurrió con Jorge Alessandri (1958-1964) hasta 1964, precisamente para asegurar estabilidad y arraigo de toda esa institucionalidad política.

MAG: Tal como señalan, el tiempo en asegurar que el significado de la elección de Alessandri en 1920 (una especie de "estallido social", pero en expresión electoral), que implicó la posibilidad de incorporación de las clases medias y parte de los sectores populares, sólo empieza a convertirse en un orden político 12 años después, pero para que empiece, y solamente empiece a conformarse un nuevo orden socioeconómico, tienes 18 años. Y eso que, como bien lo decían, quedaron problemas pendientes no sólo de la institucionalidad política sino del orden socioeconómico, como la cuestión agraria, entre otros. Hoy todo ese ese tiempo de cambio en el orden socioeconómico y político debe acelerarse y sin ruptura del orden político.

Es difícil que la institucionalidad que salga del actual proceso constituyente no sea legítima. Lo interesante es que esta vez la legitimidad será doble: una, por la participación y dos por el resultado. Hay que pensar que un resultado 
enteramente distinto a las demandas del estallido, un rechazo, independientemente de su legitimidad legal, tendrá un problema de legitimidad valórica. Por ende, el tema de la participación y el tema del contenido en el proceso constituyente son fundamentales en la legitimación, como lo fueron en la elección de convencionales, en la que la diversidad de quienes fueron electos expresaba, como hemos dicho, la diversidad del estallido y en la que los partidos ganadores eran los que estuvieron cercanos al estallido y al mismo tiempo optaron por el cambio constitucional.

\section{AO: ¿Y qué ocurre con los sectores de oposición críticos de los contenidos de la nueva Constitución?}

MAG: La legitimidad de todo el proceso planteada, a mi juicio, tiene como base principios de transformación que yo creo son bastantes sustantivos, entre ellos: plurinacionalidad, paridad de género en todos los ámbitos, prioridad de derechos humanos y sociales garantizados efectivamente, régimen político participativo que permita expresión y gobierno efectivo de mayorías, modelo de desarrollo sustentable ambiental y socialmente, con rol dirigente y protector del Estado, descentralización efectiva. Por cierto, existe una derecha que intentará oponerse. Aunque creo que se va a producir una disociación entre derecha y mundo empresarial. Ese mundo empresarial va a verse obligado a conversar directamente y no a través de los representantes de la derecha, que representa muy poco hoy día. Entonces va a tener que existir la capacidad, en la Convención Constitucional y en el próximo gobierno, de conversar y hacer ver al mundo empresarial algunos pilares fundamentales en lo que esto se va a basar y que ese mundo deberá aceptar, además de la reforma tributaria y el estricto cumplimiento de las cargas tributarias: mayor intervención del Estado, dominio y control público de los recursos naturales, en que los servicios sean fundamentalmente públicos y los servicios privados subordinados al público; un Estado plurinacional en el que se resuelve el problema de integración de los pueblos originarios que tiene que ver con las tierras, lo que sabemos está relacionado con el problema de las empresas forestales y de grandes grupos económicos; las AFP's ${ }^{5}$ van a desaparecer, quizás de manera gradual, pero van a desaparecer. Tendrá que ser el pueblo elegido en la Convención el que dialogue y plantee las exigencias al sector empresarial y en ese sentido va a tener una enorme legitimidad.

El gran problema, que se conecta con el primer tema de la conversación, con la transformación de la sociedad contemporánea, con la crisis medioambiental, que es una "crisis civilizatoria", es el del modelo de desarrollo global fracasado. Lo que se debe considerar es que la Constitución no sólo repara problemas del pasado, sino que permite pensar y asumir los problemas del futuro. Y el tema del modelo de desarrollo es un tema crucial, frente al cual no sabemos mucho cómo se debe asumir y por lo tanto lo importante es generar los mecanismos que permitan ir transformando el actual hacia un nuevo modelo. No va a ser el modelo de tal o cual otro país. Además, la implementación de todas estas cuestiones fundamentales, más allá de los principios constitucionales que las rijan, no depende del texto constitucional, sino que se define en los programas de gobierno. Por eso es crucial que lo que se vaya decidiendo y aprobando en la Convención Constitucional, pueda ser implementado en el próximo gobierno y Parlamento que se elige en noviembre de 2021.

\section{Bibliografía surgida en conversación}

Arendt, H. (2001). La condición humana. Barcelona: Paidós.

Garretón, M. A., (2016) “La ruptura entre política y sociedad. Una introducción”. En M.A. Garretón (ed.), La gran ruptura. Institucionalidad política y actores sociales en el Chile del siglo XXI. Santiago: LOM Ediciones, 7-20.

Garretón, M.A. (2021) “Reflexiones sobre movimientos sociales, estallido y proceso constituyente”. En M.A. Garretón (coord.), Política y movimientos sociales en Chile: Antecedentes y proyecciones del estallido social de octubre 2019. Santiago: LOM Ediciones - Fundación Friedrich Ebert-Chile.

Garretón, M.A. (2021a). "Del estallido social al proceso refundacional. El nuevo escenario de la sociedad chilena”. Asian Journal of Latin American Studies, 34 (2), 39-62. https://doi.org/10.22945/ajlas.2021.34.2.39

Osorio Rauld, A. y Reyes, V. (2021). “Among Uncertainty, Fear and Reluctance to Change: The Basis of the 'Rejection' to the New Constitution in Chile's Big Business Before the Social Outbreak of 2019”. Journal of Applied Business and Economics, 23 (6), 171-183.

5. Las AFP o Administradoras de Fondos de Pensiones, son instituciones privadas de capitalización individual que operan en Chile para administrar los fondos de los cotizantes, ante la ausencia de un sistema de pensiones con solidaridad. 